\title{
Do McCollough effects provide evidence for global pattern processing?
}

\author{
CELESTE MCCOLLOUGH \\ USAF Research Laboratory, Mesa, Arizona
}

\begin{abstract}
Contingent color aftereffects (CAEs, or McCollough effects) were induced using two pairs of orthogonally related patterns (horizontal/vertical and concentric/radial) to determine whether the CAEs of the four patterns are independent. Tests using composite test patterns (like those employed by Emerson, Humphrey, \& Dodwell, 1985) suggested independent aftereffects. However, tests using unitary patterns indicated additive or competing effects of the four patterns in regions where line orientations were similar, and tests isolating such regions showed clear interactions between the pattern aftereffects. The results fail to support the claim that global (rather than local) features of the patterns control these CAEs.
\end{abstract}

Color aftereffects contingent on grating orientation can be readily induced in most observers. To induce them, square-wave gratings of orthogonal orientation (usually horizontal and vertical; see Figure 1a) are viewed alternately in complementary colors (usually green and magenta) for several minutes. The resulting contingent color aftereffects (CAEs) are negative; after viewing green horizontal and magenta vertical gratings, horizontal white lines chalked on a blackboard appear pinkish and vertical lines appear greenish.

Like negative afterimages (AIs), CAEs occupy a region of visual space defined by retinal rather than geographical coordinates; the reference point for retinal coordinates is the fixation point. CAEs and AIs are observed only in the retinal region that has been exposed to the inducing stimulus. But unlike a negative AI, a CAE is an aftereffect in which opponent colors can be seen successively at the same retinal location, contingent on the pattern reaching the retina from a test stimulus. A black-and-white grating looks pale magenta (pink) when the lines are horizontal; if the pattern is rotated $90^{\circ}$, the grating looks pale green. On patterns different from the inducing patterns (such as those in Figures $1 \mathrm{~b}$ and $1 \mathrm{c}$ ), pink is seen wherever the lines are approximately horizontal and green is seen wherever they are approximately vertical; regions of oblique lines appear colorless. Thus, the CAE shows that different colors can be perceived at the same retinal location when only pattern has changed; composition of the light reaching the retina remains the same.

This research was supported under Contract No. F33615-90-C-0005 between the United States Air Force and the University of Dayton Research Institute. Figures were prepared in final form by Curtis Sayers under Contract No. F41624-97-D-5000 between the USAF and Hughes Training, Inc. Programming support by Steve Riedler and helpful comments from Jack Broerse, Julie Lindholm, Michelle Bieber, and Jack Werner are gratefully acknowledged. Portions of this paper were presented at the annual meeting of the Association for Research in Vision and Ophthalmology, Sarasota, FL, 1994. Correspondence should be addressed to C. McCollough, 6030 S. Kent, Mesa, AZ 85212-6061 (e-mail: celeste@getnet.com).

\section{Local and Global Features}

CAEs were first reported by Kohler (1951) as "phantom color fringes" observed at the right and left edges of bright rectangles (such as windows) following adaptation to prism glasses. The first report of McCollough effects (McCollough, 1965) grew out of the finding that populations of single cells in the primary visual cortex are selectively sensitive to left- or right-facing vertical edges (Hubel \& Wiesel, 1962). Since such populations of cells were also selective for the orientation of edges in the frontoparallel plane, McCollough regarded CAEs contingent on grating orientation as being essentially similar in their origin to CAEs contingent on polarity, and she argued that both could be due to color biasing or adaptation of cortical cells selective for particular kinds of edges. That is, coloring the white bars in a vertical grating would in fact give the same color biases to cells selective for left-facing and right-facing edges at that orientation.

The simple cortical cells identified by Hubel and Wiesel (1962) have small receptive fields. If they play an important role in generating CAEs, then CAEs must be dependent on line orientation in a relatively local part of a pattern, the part that falls within the receptive field of a V1 cell selective for orientation. Moreover, CAEs require the local luminance contrast that defines an edge; reducing luminance contrast decreases the aftereffects (Ellis, 1977; Mikaelian, 1980). Thus, local orientation of high contrast edges has been widely regarded as central to the explanation of these aftereffects.

However, many patterns have both local and global features. It is of great theoretical importance to know whether CAEs are dependent in any way on the context in which the local line segment appears. Does the total Gestalt make any difference? Perhaps. Emerson, Humphrey, and Dodwell (1985) reported that they were able to obtain CAEs to each of the pairs of induction gratings in Figure 1, using different groups of subjects. They further reported that they were able to get independent CAEs to each of the three pairs of gratings when the same subjects were given successive inductions with each of the three pairs of fig- 
(a)

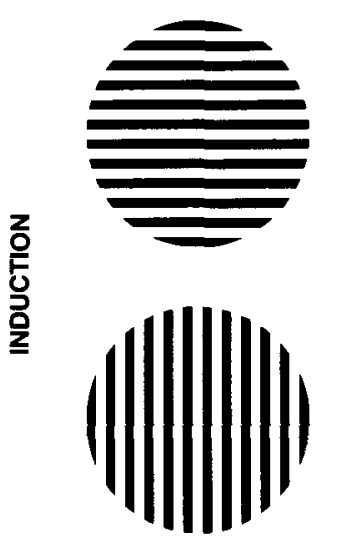

(b)
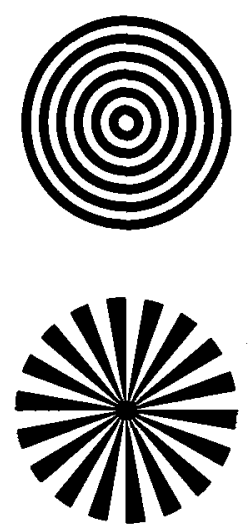

(c)
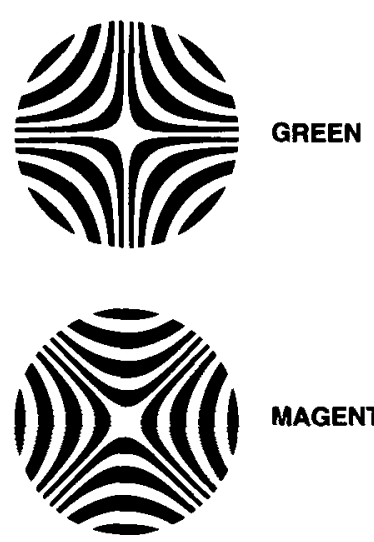

MAGENTA
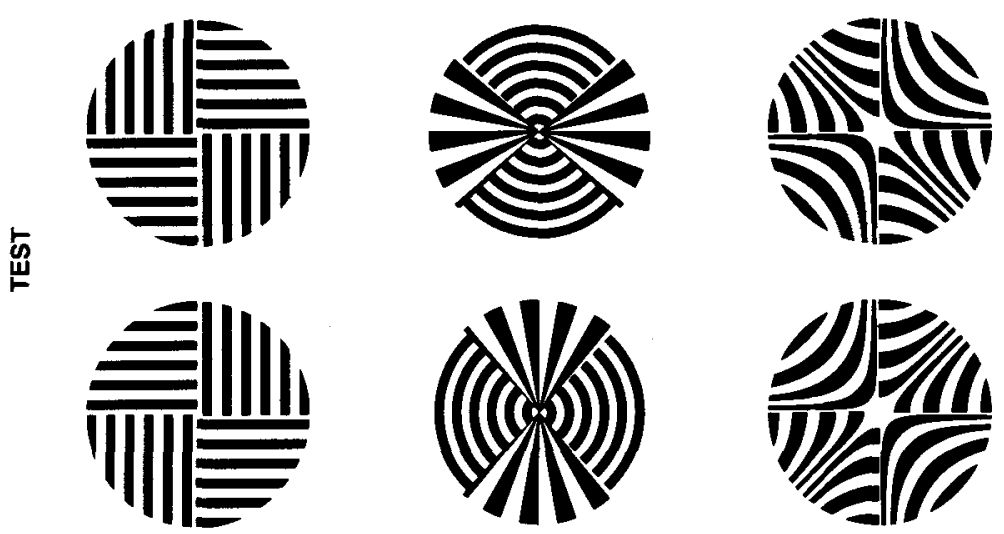

(1)


(2)

Figure 1. Induction and test patterns redrawn from Emerson, Humphrey, and Dodwell (1985). These patterns fall into orthogonal pairs designated, from left to right, as (a) Cartesian, (b) polar, and (c) hyperbolic gratings. Emerson et al. balanced the induction colors across observers. The induction colors represented in this figure are green for the upper and magenta for the lower member of each induction pair. Note-From "Colored Aftereffects Contingent on Patterns Generated by Lie Transformation Groups," by V. F. Emerson, G. K. Humphrey, and P. C. Dodwell, 1985, Perception \& Psychophysics, 37, p. 157. Copyright 1985 by the Psychonomic Society, Inc. Adapted with permission.

ures. The implications of their experiment can be made clear from an example.

Figure 1 specifies a particular set of induction colors: green horizontal and magenta vertical; green concentric and magenta radial; green $0 \% 90^{\circ}$ hyperbolas and magenta $45^{\circ} / 135^{\circ}$ hyperbolas. Three of these patterns (horizontal grating, radial segments, and $0^{\circ} / 90^{\circ}$ hyperbolas) have horizontal lines in the region left of center; two of them are shown as having green induction, and one (radial) is shown as having magenta. If an observer, fixating at the center of black-and-white prints of these three patterns, reports green CAEs on these horizontal lines in the radial segment pattern but pink CAEs in the same area on the other two patterns, then the CAEs are clearly de- pendent on the global context in which the locally horizontal lines are placed. Such a result would mean that two opponent colors can be seen successively in the same retinal region (the region left of fixation), despite the presence of the same local feature (horizontal line segments). In other words, it would show that global pattern features can bring about different aftereffect colors in the same retinal region, even when the local pattern feature is unchanged.

\section{Theoretical Importance}

Evidence for a global feature effect is crucial for the "functional" theory of CAEs offered by Dodwell and Humphrey (1990), who argue that visual elements sensi- 
tive to spatial frequency, responding to orientation of an edge in a particular location, also respond to "properties in the 'neighborhood' of that edge."

Evidence for a global effect also has implications for many of the other theorists who explain CAEs in terms of visual psychophysiology (e.g., Breitmeyer \& Cooper, 1972; Held \& Shattuck, 1971; Jameson, Hurvich, \& Varner, 1979; Lovegrove \& Over, 1972; Stromeyer \& Dawson, 1978; Viola, 1966; Watanabe, 1995; White, Petry, Riggs, \& Miller, 1978). Such evidence would indicate involvement of cortical cells with receptive fields considerably larger than those of $\mathrm{V} 1$ cells responsive to spatial frequency and orientation. Cells with a more global response pattern have actually been found in area V4 of macaque visual cortex. Gallant, Connor, Rakshit, Lewis, and van Essen (1996) reported that $17 \%$ of 103 cells tested in that area gave significantly larger responses to the most effective polar (Figure 1b) or hyperbolic (Figure 1c) grating than to any Cartesian grating (Figure $1 \mathrm{a}$ ). There were no cells that gave significantly larger responses to Cartesian gratings than to the other two grating classes, and the responses obtained with polar or hyperbolic gratings generally could not be predicted from the cell's response to Cartesian gratings.

Explanation of CAEs in terms of visual psychophysiology has recently been clarified and extended to biophysical optics. Several studies (Broerse \& O'Shea, 1995; Broerse, Vladusich, \& O'Shea, 1999; Pieper, 1995) have revisited the distinction between CAEs involving differences in edge polarity and those involving differences in orientation, noting that edges of identical orientation but different polarity can be paired either with the same colors (e.g., red left-facing and red right-facing edges) or with complementary colors (red left-facing and green right-facing edges). In keeping with McCollough's (1965) original analysis, CAEs induced by a uniformly colored grating at a particular orientation (as in McCollough's original demonstration) are similar to CAEs induced by a grating in which colored fringes are simulated on leftand right-polarity edges (i.e., only the edges themselves are color-biased). However, Pieper (1995) has shown that when vertical gratings have red-fringed left-facing and green-fringed right-facing edges during induction, the CAEs are limited to fringe colors (green on left-facing, red on right facing edges). His finding fits nicely with the demonstration by Broerse et al. (1999) that CAEs induced with red fringes on both left- and right-facing edges of vertical gratings involve two components: fringe color that is independent of bar width, and spread color that is increasingly visible with decreasing bar width. These two components can also be identified when induction is with traditional red vertical and green horizontal gratings, provided that the width of white bars is varied in test patterns. Broerse et al. (1999) propose that the fringe color component serves to neutralize naturally occurring chromatic aberration at high-contrast edges, whereas the spread color component is related to neon color spreading. This proposal clearly involves local rather than global factors.
Finally, evidence supporting a global effect will be of interest to those arguing for or against the major alternative theory of CAEs, a theory that bypasses visual psychophysiology and explains the aftereffects as learned (conditioned) perceptual responses (Allan \& Siegel, 1986, 1993, 1997a, 1997b; Allan, Siegel, \& Linders, 1992; Murch, 1976; Siegel \& Allan, 1992; Siegel, Allan, \& Eissenberg, 1992). Recent controversy between proponents and opponents of the conditioning theory has concerned CAEs contingent on form; this dispute hinges in fact on the local-global issue. An early study (Foreit \& Ambler, 1978) failed to obtain CAEs following induction with a magenta outline square and a green cross, but a study with similar induction figures (Siegel et al., 1992) did obtain CAEs strong enough to be measurable with a variant of the method of constant stimuli. Two other replications of the same study (Broerse \& Grimbeek, 1994; Humphrey, Herbert, Symons, \& Kara, 1994) indicated that CAEs to such forms could be obtained only when eye movements were restricted, so that particular elements of the forms (vertical or horizontal lines) always had the same induction color in any local region of the retina. Broerse and Grimbeek argued that the CAEs obtained in such cases are dependent on local retinal components, not on the more global pattern itself. Advocates of the conditioning explanation (Allan \& Siegel, 1997b; Siegel, Allan, \& Eissenberg, 1994) agree that induction with strict fixation will produce CAEs interpretable in terms of local (retinotopic) factors, but they contend that CAEs dependent on global (spatiotopic) factors can be obtained with nonfixating subjects. A similar controversy over CAEs contingent on text (Allan, Siegel, Collins, \& MacQueen, 1989; Humphrey, Skowbo, Symons, Herbert, \& Grant, 1994) also remains unresolved.

Other associative accounts of CAEs (Barlow, 1990; Bedford, 1995, 1997) deal with correlation and decorrelation of entire perceptual dimensions. It is not clear how evidence for global factors might affect these accounts.

\section{Experiments Suggesting a Global Effect}

Some earlier experiments have a bearing on the local-global question. Riggs (1973, 1974), using the patterns shown as in Figure 2, found a CAE that he believed was "strongly dependent on radius of curvature" and not explainable in terms of tilt or orientation. His conclusion that "curvature is a specific feature of human visual perception" is an early statement in favor of global pattern features (cf. Crassini \& Over, 1975; White \& Riggs, 1974). But Stromeyer (1974a) found that this CAE changed color with shifts in fixation and argued that it could be explained in terms of local straight-line effects. MacKay and MacKay (1974) failed to find a curvature CAE when they required their observers to make eye movements along the chord of the adaptation arc during induction. Moreover, Sigel and Nachmias (1975) found more saturated CAEs near the edges of Riggs's patterns, where line orientation differences are maximal, than at the center, where lines are horizontal in both induction patterns. 
Inspection

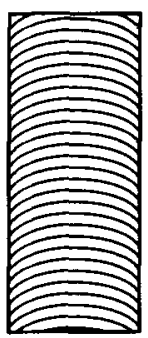

Ped

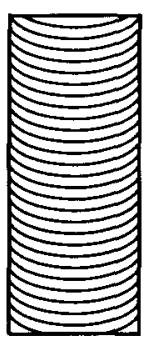

Green
Test

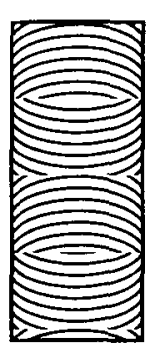

Achromatic
Figure 2. Induction and test figures redrawn from Riggs (1973). Note-From "Curvature as a Feature of Pattern Vision," by L. A. Riggs, 1973, Science, 181, p. 1070. Copyright 1973 by the American Association for the Advancement of Science. Adapted with permission.

Crassini and Over (1975) studied curvature aftereffects with curved inspection figures that differed in degree rather than direction of curvature and reported that the CAEs were often "partial"- that is, restricted to limited regions of the test arcs. Thus, none of these experiments on curvature has provided clear evidence for a global effect.

Cavill and Robinson (1976) used concentric circles and radial lines (Figure 3a) as induction and test patterns. Verbal reports indicated the expected aftereffects, but the experimenters recognized that local line orientation could produce such aftereffects if observers tended to fixate the center of the induction patterns. In that case, "every point of the visual field is stimulated by lines in the two patterns which are orthogonal." Using similar figures (Figure 3b), Yasuda (1978) recorded eye movements during induction and found that "the fixation points were apt to concentrate on the center of the patterns in spite of the free scanning instruction" (p. 119). When he required observers to move fixation at $1-\mathrm{sec}$ intervals during the induction period, only 2 of 7 observers saw any CAEs, and the colors they saw were very faint, could not be fixated, and appeared "at only one part of three sectors of the compound test patterns." Therefore, merely finding that CAEs occur with polar grating induction does not make the case for a global effect. ${ }^{\prime}$

However, the successive induction procedure of Emerson et al. (1985) could make such a case. Because these experimenters used more than one pair of mutually orthogonal patterns successively with the same observers, the locus of fixation is not an issue. As the argument above (based on Figure 1) has shown, these successive inductions could, in principle, give evidence that CAEs of different colors can be induced in the same retinal area with the same local line orientation, contingent on the global context in which those lines are placed. But the Emerson et al. report does not actually provide this evidence.

After induction, their subjects looked successively at each of the six test patterns shown in the lower half of Figure 1 and fixated the center of the pattern. They then indicated the colors seen on these patterns by drawing out- lines on photocopies of those patterns and writing a color name within the outline. Their drawings were scored as having the expected complementary color (scored positive) or another color (scored negative) and as being either "complete" or "partial." If an observer outlined in a test pattern both quadrants that came from a single induction pattern, the response was scored "complete." Outlines of only one quadrant, or only parts of one or both quadrants, were scored "partial." Five of their 12 subjects reported at least one "complete" CAE from each pair of test patterns (Figure 1, bottom), and 8 of the 12 reported CAEs from at least two of the three pairs.

These quantitative data are offered in support of the claim that some subjects acquired independent CAEs to two or three of the pattern pairs. However, the data reported are consistent with another hypothesis-namely, that some subjects acquired line-dependent CAEs to two or three of the pattern pairs and that these CAEs interacted in local areas, sometimes reinforcing each other additively and sometimes canceling each other. To understand this hypothesis, consider the interaction of induction conditions $a$ and $b$ in Figure 1, where both horizontal $(H)$ and concentric $(C)$ gratings are green, and vertical $(V)$ and radial $(R)$ gratings are magenta. Since the concentric line segments in test pattern bl are approximately horizontal, the pink CAEs for horizontal lines may reinforce the pink CAEs for concentric circles in this test pattern-an additive effect. In the same test pattern, the pink CAEs for horizontal lines may cancel the green CAEs for the (approximately horizontal) radial segments-a subtractive effect. Green CAEs for vertical lines may similarly reinforce green CAEs for radial segments in test pattern b2 and cancel pink CAEs for concentric circles. Thus, the data reported by Emerson et al. (1985) could have arisen from local line CAEs that, in some test patterns, interacted additively and reinforced each other.

These quantitative results also do not reveal whether any subject saw CAEs of different colors on lines of the same orientation and imaged on the same retinal area when the global context differed. Nevertheless, the experiment may have contained important evidence on this point. There seem to have been cases where,

for example, a horizontal contour in one part of a particular pattern (say, near the left edge) would be reported as having an appropriate pinkish tint if it was part of a horizontal grating or the $0 \% 190^{\circ}$ hyperbolae, but an appropriate green tint if it formed part of the radial pattern. (Emerson et al., 1985, p. 160)

This led Emerson et al. to conclude that some of the CAEs were "pattern-specific in the sense that (the) hue is determined by the context of the whole pattern rather than the local contour orientations alone." Unfortunately, the authors do not say how often such reports occurred.

\section{Going Forward From the \\ Emerson et al. (1985) Experiment}

The successive induction procedure can yield more information on this important point when additional kinds 
(a)
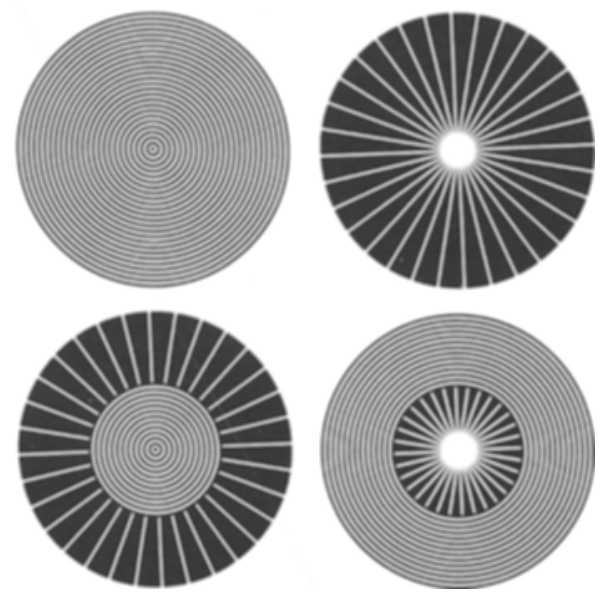

(b)
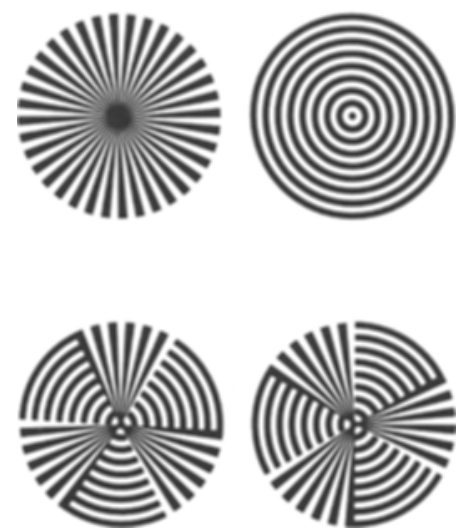

Figure 3. Induction figures redrawn from (a) Cavill \& Robinson (1976) and (b) Yasuda (1978). Note- (a) From "A Color Aftereffect Contingent on Complex Pattern Features," by J. Cavill and J. O. Robinson, 1976, Perception \& Psychophysics, 19, p. 455. Copyright 1976 by the Psychonomic Society, Inc. Adapted with permission. (b) From "Color Aftereffects Contingent on MacKay Complementary Regular Patterns," by K. Yasuda, 1978, Japanese Psychological Research, 20, p. 118. Copyright 1978 by the Japanese Psychological Association. Adapted with permission.

of data are collected. For example, test patterns could include some copies of the induction patterns. If global factors are operative, presentation of the whole pattern (e.g., concentric circles) should favor a CAE that is determined globally by the corresponding induction pattern, and some observers should report a "complete" CAE that fills the entire pattern even though they have also received the Cartesian induction. If local factors are adding algebraically, on the other hand, such "complete" CAEs should rarely or never occur, and there should be many reports of "partial" CAEs with different colors in different regions of the test pattern. The experimental design described below included whole ("unitary") test patterns identical to the four induction patterns as well as the "composite" test patterns used by Emerson et al. (1985).

If there are local additive and subtractive effects of the multiple inductions, it will be helpful to have a quantitative (colorimetric) measure of CAE strength in addition to the subjects' verbal reports. As the example in Figure 4 shows, local line effects could be additive on some test patterns and subtractive on other test patterns. Each test pattern from Figure 4 can be presented on a CRT, and subjects can be asked to adjust its color so that the pattern appears achromatic. Such chromaticity adjustments provide a sensitive measure of CAE strength (MacKay \& MacKay, 1973; Riggs, White, \& Eimas, 1974). If CAEs are not larger on additive patterns than on subtractive patterns, the case for independent CAEs and global effects will be strengthened. Therefore, the experimental design included colorimetric measurements for quantitative analysis of the CAEs seen on such patterns.

\section{METHOD}

\section{Subjects}

Eight observers ( 3 women, 5 men) were recruited from laboratory staff members, friends, and family. All had $20 / 20$ or better visual acuity, and all had error scores within the range of average color-discriminating ability on the Farnsworth-Munsell 100-Hue Test. None of the observers had previously served in CAE experiments; all agreed to come for a screening session and four experimental sessions. The screening session included 45 color-matching trials to introduce the test patterns and to provide experience with small color differences in this region of color space.

\section{Design}

The experiment was limited to the Cartesian and polar induction patterns represented in Figure 4. Colorimetric data were collected and analyzed according to a three-factor within-observers analysis of variance (ANOVA) design with repeated measures (see Table 1). Two observers were assigned to each of the four possible combinations of induction pairs (Cartesian, polar) and induction colors (green, magenta). The eight test patterns shown in Figure 4 were created because unitary test figures did not appear uniformly colored after induction. These eight patterns became additive or subtractive, depending on the observer's induction conditions. Thus, for each observer, each of the eight cells of the induction pair factor in Table I contained one of these eight patterns, and each of the eight patterns was additive for half the observers and subtractive for the other half. Similarly, for any given pattern, the expected $\mathrm{CAE}$ color was green for half the observers and pink for the other half. Figure 4 and Table 1 show the actual conditions for one of the four groups. As a control, a ninth test pattern was included; it contained a single unpatterned (blank) quadrant to the right of fixation.

Each pattern was presented on a monitor screen in an initial color whose chromaticity coordinates varied at random between pale green and pale magenta, along a line passing through D65 white (Figure 5). The observer's task was to vary the color, using a computer 
Induction Patterns



Test Patterns with Additive CAEs
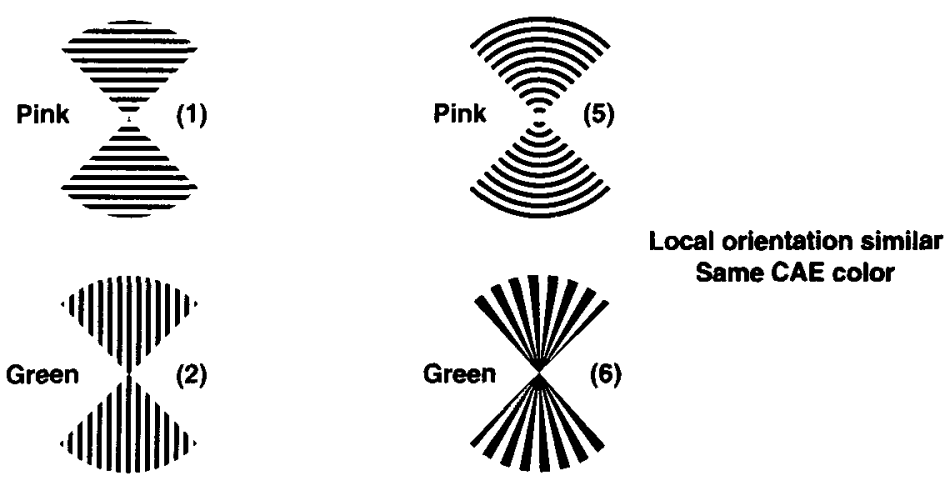

Test Patterns with Subtractive CAEs

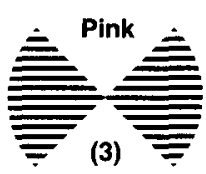

(3)<smiles>C#CC#CCCC#C</smiles>

(4)

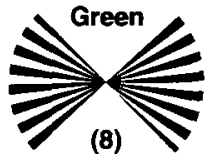

Local orientation similar Different CAE colors

Figure 4. Diagram representing CAEs induced by green horizontal, magenta vertical, green concentric, and magenta radial gratings. The expected CAEs will be additive for the test patterns in the middle two rows, since the concentric $(C)$ grating coincides in part with the horizontal $(H)$ grating in these sectors, while the radial ( $R$ ) grating coincides in part with the vertical (V) grating. The CAEs will be subtractive for the remaining test patterns, where $C$ has more local similarity to $V$ than to $H$, and $R$ has more local similarity to $H$ than to $V$. The eight test patterns are distinguished by numbers.

pointing device (mouse), until the pattern appeared achromatic (neither pink nor green). When unitary patterns like the induction patterns were tried, no setting could be found that appeared achromatic throughout. Achromatic settings were possible with the Figure 4 patterns, although slight differences in CAE strength were often visible within them. During this task, the test pattern appeared repeatedly for $4 \mathrm{sec}$, with a 4 -sec dark period between presentations, to minimize local color adaptation and Als. The observer viewed as many repetitions of the pattern as necessary, signaling by mouse key when the setting was satisfactory. Before induction, the observer made five such settings for each of the nine patterns, in a random order; these settings are the pretest data. Following induc- 
Table 1

Design for Colorimetric Data

\begin{tabular}{|c|c|c|c|c|c|c|c|c|}
\hline \multirow{3}{*}{$\begin{array}{l}\text { Factors } \\
\text { Local CAE relation } \\
\text { Expected CAE color }\end{array}$} & \multicolumn{8}{|c|}{ Levels } \\
\hline & \multicolumn{4}{|c|}{ Additive } & \multicolumn{4}{|c|}{ Subtractive } \\
\hline & \multicolumn{2}{|c|}{ Green } & \multicolumn{2}{|c|}{ Pink } & \multicolumn{2}{|c|}{ Green } & \multicolumn{2}{|c|}{ Pink } \\
\hline Induction pair & $V(2)$ & $R(6)$ & $H(1)$ & $C(5)$ & $V(4)$ & $\mathrm{R}(8)$ & $H(3)$ & $C(7)$ \\
\hline
\end{tabular}

Note-Induction pairs and test pattern numbers are given as in Figure 4.

tion, the procedure was repeated as a posttest. The difference between pretest and posttest means indicates the amount of CAE present after induction.

An experimental session also included a pretest and posttest with black-and-white printed test patterns, matching the induction patterns in spatial frequency and displayed one at a time in overhead illumination. Four were unitary patterns like the induction patterns, printed as negatives from the monitor patterns (black stripes or sectors on a white background instead of white stripes or sectors on a black background). Four additional composite patterns, like those used by Emerson et al. (1985), were created on the monitor and printed as hard copies. Two composite patterns were made up of alternate $\mathrm{H}$ and $\mathrm{V}$ sectors, separated along the horizontal and vertical axes of the figure. The other two composite patterns were made up of alternate $\mathrm{C}$ and $\mathrm{R}$ sectors, separated along the diagonals at $45^{\circ}$ and $135^{\circ}$. The $\mathrm{C}$ and $\mathrm{R}$ composites contain sectors that can be designated "additive" or "subtractive," as illustrated in Figure 4. Because the H and V composites are separated along the $90^{\circ}$ and $180^{\circ}$ meridians, each sector is aligned with $\mathrm{C}$ at one edge and with $\mathrm{R}$ at the other; these composites do not fall clearly into additive or subtractive categories.

An eight-page "coloring book" was provided; its pages were the same size as the pages on which the hard-copy patterns had been printed, and each page contained an outline circle of the same size as the pattern. Within the booklet, the four unitary patterns appeared first, in the order $\mathrm{C}, \mathrm{V}, \mathrm{R}, \mathrm{H}$. The remaining four pages contained outline circles for the composite patterns, with dividing lines separating the four sectors. ${ }^{2}$ The observers looked at the printed test patterns, one at a time, with fixation at the center of the pattern, then selected soft pastel pencils to reproduce what they had seen within the outlines on the coloring book page. These responses were later scored by the Emerson et al. criteria as "partial" or "complete" and positive or negative.

In short, the design included (1) colorimetric measurements (a color cancellation task) performed as the pretest and again as the posttest in a fully balanced within-subjects ANOVA and (2) sketches made by the observers of the colors they saw on printed patterns. Each observer completed this design in a 1 -h experimental session, then repeated the same design each day for 3 more days.

\section{Apparatus}

Induction and test patterns $\left(12 \mathrm{~cm}\right.$ and $12^{\circ}$ diameter) were presented on a Barco Calibrator monitor controlled by an IRIS 4D70 graphics workstation. The monitor was placed at the back of a black plywood box, $54 \times 55 \times 68 \mathrm{~cm}$ deep. The patterns were drawn at the center of the display and viewed through a $25-\mathrm{cm}$-diameter opening in an opaque screen. Another screen with a $10 \times 10 \mathrm{~cm}$ opening was placed at the front of the box, and a chin-and-forehead rest just behind this screen served to stabilize the observer's head position at approximately $57 \mathrm{~cm}$ from the display. The room was almost totally dark while the display was in use. Luminance was $49 \mathrm{~cd} / \mathrm{m}^{2}$ for both magenta $\left(u^{\prime}=.2827, v^{\prime}=.3625\right)$ and green $(.1311$, $.5621)$ stripes in the induction patterns. Test pattern stripes were presented at a lower luminance $\left(12 \mathrm{~cd} / \mathrm{m}^{2}\right)$, since previous studies have shown that CAEs are stronger with high-luminance induction and low-luminance testing (Stromeyer, 1974b; White, 1976).

\section{Induction Procedure}

The four induction patterns were presented alternately during a 16-min induction period, in the sequence $\mathrm{H}, \mathrm{V}, \mathrm{C}, \mathrm{R}$. Each pattern appeared for $9.5 \mathrm{sec}$, followed by a 0.5 -sec dark period. While the observer fixated a cross at the center of the circular display area, the colored pattern was displaced slowly so that its center moved in a $0.6^{\circ}$-diameter orbit around the fixation mark. The rate of this displacement was about $0.33 \mathrm{cps}$. Direction of the rotation changed between clockwise and counterclockwise after each set of four patterns. Twenty-four sets were completed within the induction period.

As noted earlier, Emerson et al. (1985) instructed their observers to fixate the center of the induction patterns. When CAE experiments use only $\mathrm{H}$ and $\mathrm{V}$ patterns, observers are usually instructed not to fixate any part of the pattern but to scan the pattern freely. The present experiment was intended to replicate the fixation conditions of Emerson et al. However, pilot studies showed that strict fixation of these bright figures was uncomfortable and produced strong negative AIs. The slight motion imposed on our induction patterns kept fixation in the near vicinity of the pattern center and did so in a controlled manner while also making the patterns more comfortable to view.

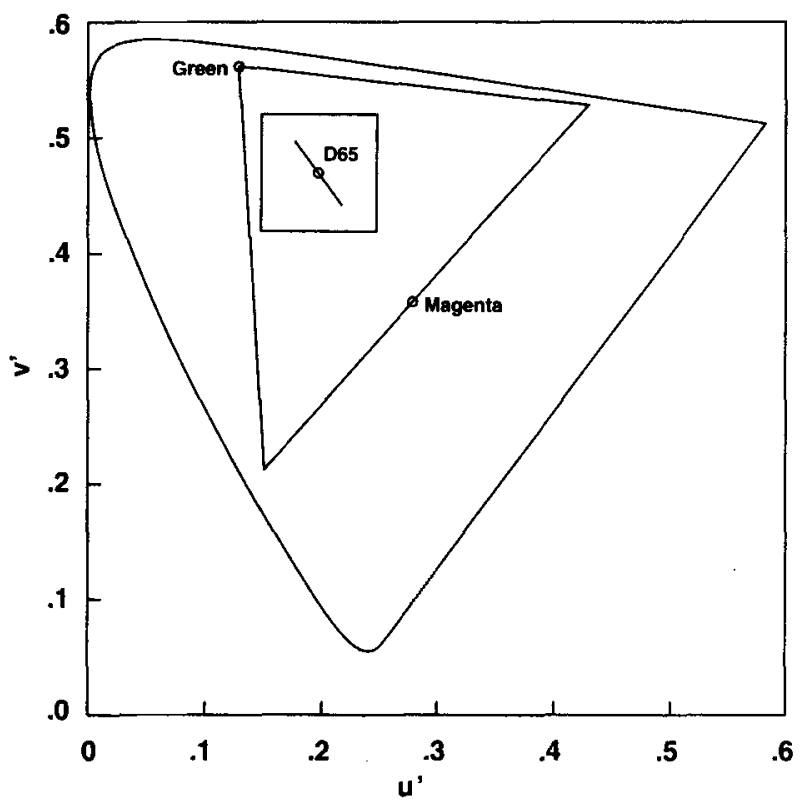

Figure 5. Induction and test colors plotted in CIE 1976 Uniform Chromaticity Space. The gamut of the Barco Calibrator monitor appears as a triangle inside the region bounded by the spectrum locus and purple boundary. The range of colors available to observers using the mouse is indicated here by a straight line passing through the daylight reference $\mathbf{D 6 5}$. 


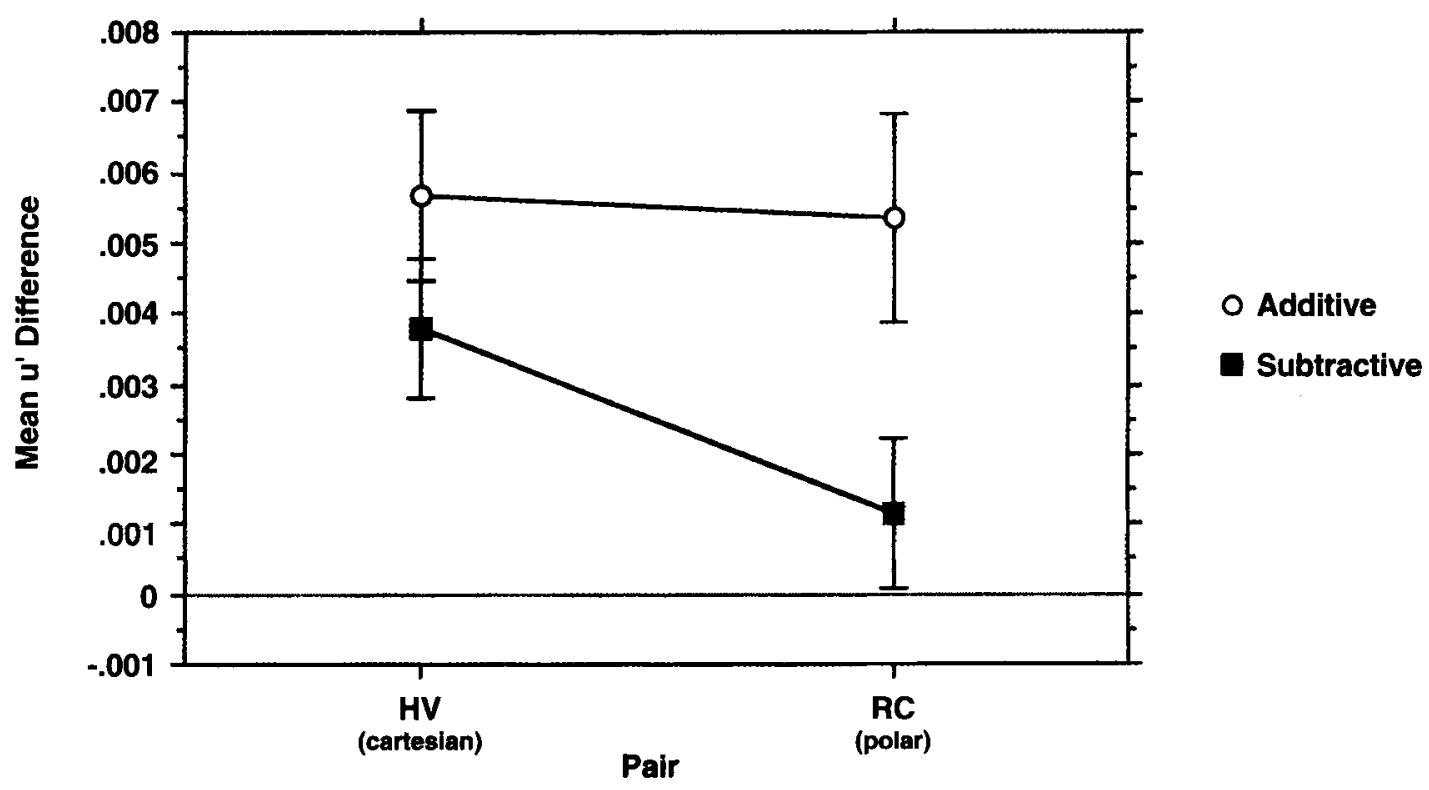

Figure 6. Interaction plot for the relation $\times$ pair effect. Mean $u^{\prime}$ difference between pretest and posttest for Cartesian and polar gratings in additive (open circles) and subtractive (filled squares) test patterns. Error bars represent $95 \%$ confidence limits.

\section{RESULTS AND DISCUSSION}

\section{Color Cancellation Data}

The $u^{\prime}$ coordinate of the observer's achromatic setting was analyzed as the dependent variable. Since all settings lay along a straight line in $u^{\prime}, v^{\prime}$ space, $v^{\prime}$ is determined when $u^{\prime}$ is known.

Each observer's five pretest settings for a given pattern at a given session were converted into a pretest mean, and the five corresponding posttest settings were converted into a posttest mean. The difference between these two means indicates the size of the CAE. When the pattern came from an induction pattern that had been presented in magenta, the CAE was expected to be green, requiring a pinker setting (higher $u^{\prime}$ value) to neutralize it. For all such cases, the difference was calculated by subtracting pretest mean from posttest mean. When the pattern came from a green induction pattern, the CAE was expected to be pink, requiring a greener setting (lower $u^{\prime}$ value), and the difference was calculated by subtracting posttest mean from pretest mean. Differences in the expected direction thus became positive in sign, and differences in the opposite direction became negative. Data from the neutral settings of the unpatterned quadrant were not included in this statistical analysis.

CAEs for additive test patterns were significantly larger than CAEs for subtractive test patterns $(F=38.1507, p=$ .0001 ), indicating that the CAEs from polar and Cartesian inductions are not independent. The advantage of additive over subtractive patterns was more pronounced for polar than for Cartesian induction patterns (Figure 6). Cartesian patterns showed stronger CAEs than polar pat- terns $(F=13.3283, p=.001)$. Emerson et al. (1985) also reported stronger CAEs to Cartesian induction patterns than to either polar or hyperbolic induction patterns. The interaction shown in Figure 6 may be explained by the greater effectiveness of Cartesian gratings.

Figure 7 presents mean $u^{\prime}$ settings from each day's pretest and posttest on each of the nine test patterns, including the unpatterned (blank) quadrant. The means are graphed separately for each relation/pair combination. All patterns representing additive relations show posttest means that clearly differ from the means for the blank quadrant. The same is true for patterns representing subtractive sectors of the Cartesian grating but not for those representing subtractive sectors of the polar grating. The Cartesian grating's CAE was still clearly present on Cartesian sectors, even when the polar induction conditions were for a CAE of opposite color. But settings for the subtractive polar sectors did not differ significantly from those for the unpatterned quadrant. It must be concluded that the observers did not see measurable CAEs on these patterns, although they should have done so if the polar and Cartesian inductions produced independent CAEs governed by the global context in which local line orientations are imbedded.

To see this point more clearly, refer again to Figure 4. The critical test patterns are those with potentially subtractive CAEs (numbered 3 and 8,4 and 7). For each of these pattern pairs, the orientation of lines at the center of each quadrant is similar, but the expected CAE color is different. The experimental question applied to pair 3 and 8 is, "Will the observers see a pink CAE in the retinal regions to the left and right of fixation when the 

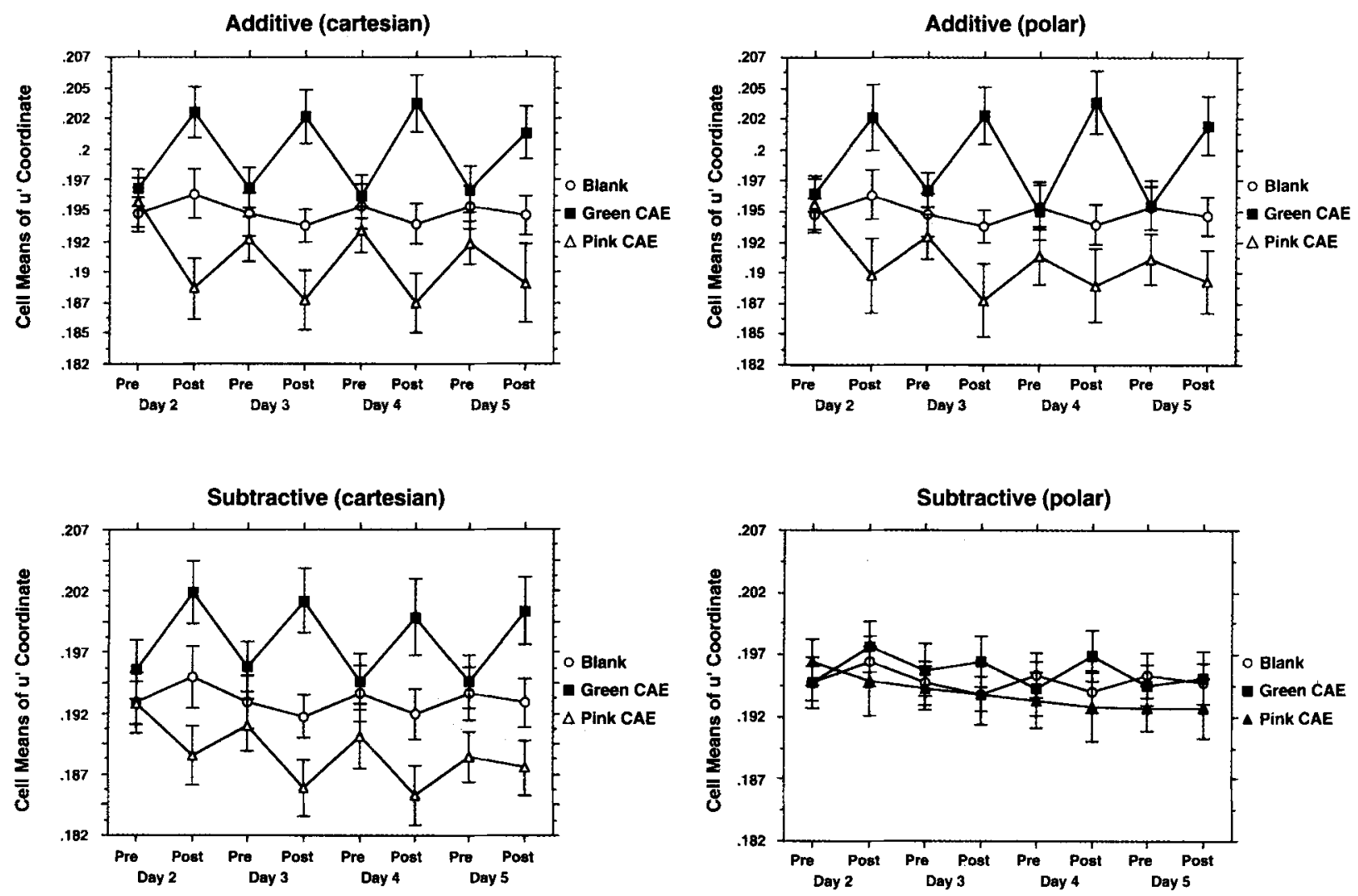

Figure 7. Group means for daily pretests and posttests for the eight additive or subtractive test patterns compared with the mean settings for the unpatterned (blank) control quadrant. Every mean is based on 40 settings ( 5 by each of the 8 observers). Open circles represent means for the blank quadrant; the same means appear in each part of the figure. Filled squares show the means for the patterns on which green CAEs are expected; triangles show those for patterns on which magenta CAEs are expected. Bars show 95\% confidence limits.

global context is 'Cartesian grating,' and a green CAE in those same regions when the global context is 'polar grating'?" The finding, shown clearly in the lower half of Figure 7, is that the pink CAE was seen in the Cartesian context, but no clear CAE was seen in the polar context. Similarly, with regard to pair 4 and 7 , observers did see a green $\mathrm{CAE}$ to the left and right of fixation when the context was Cartesian but failed to see a pink CAE in those same regions when the context was polar.

\section{Coloring Book Test}

Coloring done in the test booklets was scored by the criteria reported in Emerson et al. (1985). Each pattern component was scored as showing either "complete" or "partial" CAEs. For the four composite patterns, two responses could be scored, one for each of the two induction patterns. For the four unitary patterns, a response was scored as "complete" only if the observer colored the entire pattern. When only parts of the pattern were colored with a single color, or when different parts were given different colors, the response was scored as "partial."

Figure 8 summarizes the results for the unitary test patterns. After the first induction, no observer fully col- ored $\mathrm{H}$, and 5 observers colored part of it. By the end of four daily inductions, all but 1 of the observers had given at least one "complete" response to this pattern. V received "complete" responses from 3 observers after the first induction and "partial" responses from 2 others. No observer ever gave a "complete" response to $\mathrm{C}$, although 6 gave "partial" responses after one induction, and an additional observer gave such a response later in the study. Most of the responses to $\mathrm{R}$ were also "partial," although $1 \mathrm{ob}-$ server's responses after the second, third, and fourth inductions were scored as "complete" because he colored the entire figure except for a small region at the center.

Figure 9 summarizes the results for the composite patterns. On these patterns, which replicated the patterns used by Emerson et al. (1985), most of the responses were "complete." All observers gave "complete" responses at some time to $\mathrm{H}, \mathrm{V}$, and $\mathrm{C}$ sectors, and most of them did so after only one induction. Six observers gave at least one "complete" response to R, 5 of them after only one induction. No observer gave "partial" responses to V, and only 1 observer gave such a response to $\mathrm{H} ; 4$ observers gave "partial" responses at least once to $\mathrm{C}$, and 7 did so to $\mathrm{R}$. When only composite test patterns are used, the re- 




Figure 8. Number of observers making "complete" responses (left half) or "partial" responses (right half) to the unitary test patterns. White bars show the number of observers making such responses after a single induction (Day 1). Gray bars show the number of observers who made such responses after any (one or more) of the inductions. No "complete" responses were made to $H, C$, or $R$ on Day 1 . No "complete" responses were ever made to $C$.

sults are similar to those reported by Emerson et al.: "complete" responses far outnumber "partial" ones.

Figure 10 gives examples of responses that were scored as "partial"; induction conditions are noted beneath each drawing. Four of these examples are responses to unitary patterns. Figure $10 \mathrm{f}$ shows a "partial" response in the side sectors and a "complete" response in the upper and lower sectors. One test pattern was rotated $45^{\circ}$ on the final 2 days of the experiment; it is shown in its rotated position as Figure 10c. In this position, with its sector edges aligned with axes at $45^{\circ}$ and $135^{\circ}$, all but one observer made drawings of a "pinwheel" effect. It is clear that the rotation brought the lines of this Cartesian pattern into ori- entations that were locally similar to parts of the polar induction patterns. In the upper sector, the lines at the right came into an orientation similar to that region of the green $\mathrm{C}$ induction pattern; the CAE in that region is pink. The lines at the other side of the same sector came into an orientation similar to that region of the magenta $\mathrm{R}$ induction pattern, and the aftereffect in that region is green.

The CAEs indicated as visible on Figure 10c make several points: (1) the polar induction was effective in producing CAEs; (2) these CAEs could be seen clearly on the lines of a pattern with a different "global" context; (3) these CAEs appear to depend on similarity of local line

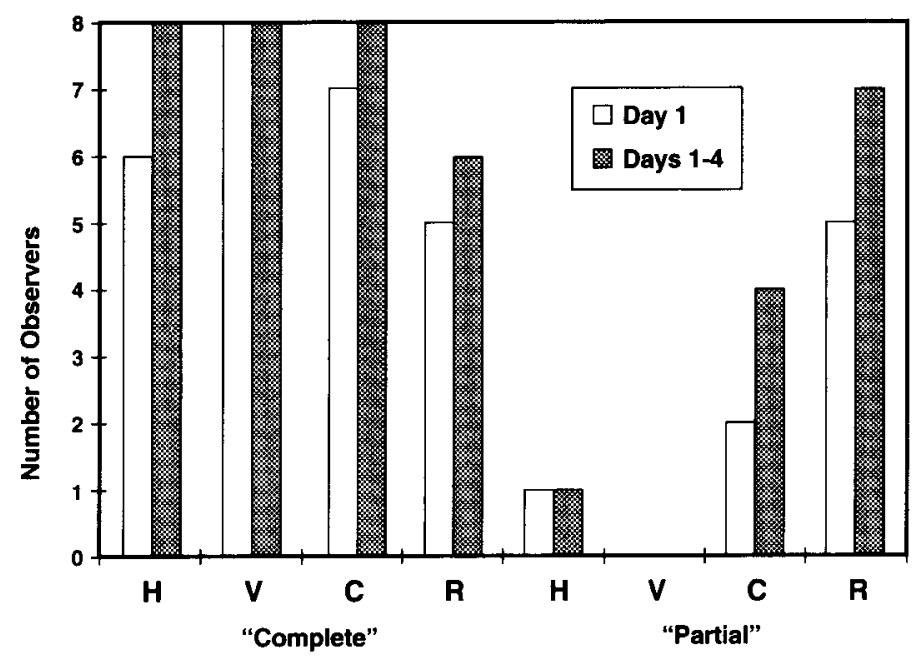

Figure 9. Number of observers making "complete" responses (left half) or "partial" responses (right half) to the composite test patterns. White bars show the number of observers making such responses after a single induction (Day 1). Gray bars show the number of observers who made such responses after any of the four inductions. No "partial" responses were ever made to $V$. 


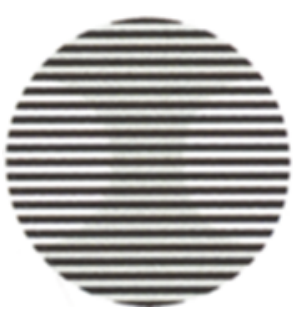

(a) Green V, R Magenta H, C

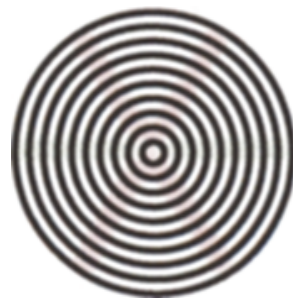

(d) Green H, C Magenta V, R

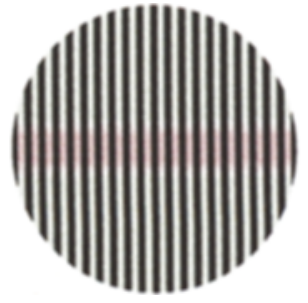

(b) Green H, C Magenta V, $R$

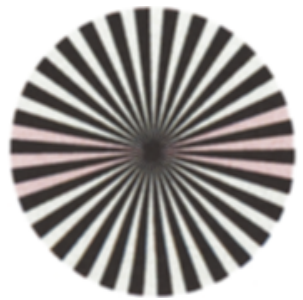

(e) Green H, C Magenta V, R

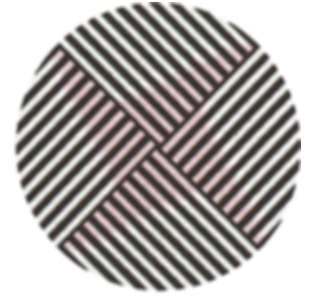

(c) Green V, C Magenta H, R

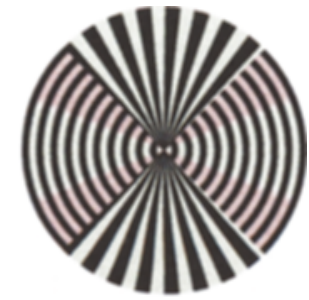

(f) Green $\mathrm{H}, \mathrm{C}$ Magenta V, $\mathbf{R}$

Figure 10. Sketches showing CAEs reported by some observers. Induction conditions are indicated below each drawing.

orientation between the test pattern and the polar induction pattern; and (4) they appear readily on diagonal straight lines where the CAEs of the Cartesian induction itself are not present (and thus do not compete with the polar CAEs).

\section{GENERAL DISCUSSION}

The results of this experiment are consistent with a view that CAEs induced by polar gratings depend on the orientation of edges in local regions of these patterns. Polar gratings can be used to induce CAEs, but their effectiveness as induction patterns does not depend on their global organization into concentric circles or radial lines.

This experiment brings two parallel lines of evidence to bear on the question of local versus global factors in CAEs. Each line of evidence leads to the same conclusion.

1. Observers' verbal reports. A great deal of information about CAEs has been obtained simply by asking observers to describe, sketch, or compare the colors that they see on test patterns presented after an induction procedure. After successive induction with both Cartesian and polar gratings, Emerson et al. (1985) found that their observers frequently saw CAEs of the appropriate colors in particular sectors of their composite test patterns. So did our observers, and they did so more often for $\mathrm{H}$ and $\mathrm{V}$ sectors ( $92 \%$ of cases) than for $\mathrm{C}$ and $\mathrm{R}$ sectors $(62.5 \%$ of cases). Considering only the polar composites ( $\mathrm{C}$ and $R$ cases), our observers filled the sectors with color in almost $75 \%$ of cases where the CAEs of polar and Cartesian induction gratings were additive; when the CAEs were subtractive, they drew such "complete" responses in fewer than half the cases.

Emerson et al. (1985) did not look at their results in terms of additive and subtractive relations. Neither did they require their observers to describe CAEs to unitary test patterns that were achromatic versions of the induction patterns. When we did so, the vast majority of responses obtained were indicative of interaction between the polarand Cartesian-induced CAEs. A unitary vertical or horizontal grating often did not appear uniformly colored; instead, a band across the center of the pattern frequently took on a hue complementary to the CAE seen on the rest of the pattern. Uniformly colored CAEs on unitary concentric or radial gratings were particularly rare, although such test patterns surely present the optimal chance for stimulating a "global vectorfield" (Dodwell \& Humphrey, 1990). Individual observers gave widely varying responses, some indicating more, and larger, areas of color than others, but only 1 observer ever came close to filling a unitary polar pattern with a single color. Humphrey (1998) reports that he also found more "partial" color responses than "complete" color responses on unitary patterns when he included such test patterns in observations revisiting the Emerson et al. study.

When the colors of all four induction patterns are taken into consideration, most of the responses found on the 256 coloring book pages could have been predicted from knowledge of the colors and orientations appearing in local regions during induction. If global factors played any role at all, that role was fully obscured by the local effects. 
2. Colorimetric data. Achromatic settings, made to cancel the aftereffect colors, showed stronger CAEs on patterns for which the polar and Cartesian inductions were additive than on patterns for which the inductions were in competition with each other. When the inductions were additive, polar and Cartesian test patterns showed almost equally strong CAEs; when the inductions were subtractive, the colorimetric settings for polar test patterns were not distinguishable from those for the unpatterned (blank control) quadrant. There is no evidence at all that the observers saw different colors in the same retinal region with the same local line orientation when the context changed from globally Cartesian to globally polar.

Finally, in weighing this evidence for interaction (and against independence) of polar and Cartesian CAEs, it should be remembered that our test patterns for colorimetric measurement were chosen because unitary test figures did not appear uniformly colored after induction. Figures 10b, 10d, and 10e illustrate typical aftereffects seen on unitary patterns.

Both lines of evidence are in good general agreement with previous studies supporting local line orientation as a major determiner of CAEs. In particular, a study of motion-contingent CAEs that employed moving polar and Cartesian gratings (Broerse, Shaw, Dodwell, \& Muir, 1994) obtained results closely paralleling those of the present study. In Broerse et al.'s (1994) Experiment 4 , subjects were induced with red vertical gratings moving left, green vertical gratings moving right, red concentric gratings expanding, and green concentric gratings contracting. Subjects then viewed four types of unitary figures in motion: concentric circles expanding, concentric circles contracting, vertical lines moving left, and vertical lines moving right. The unitary figures did not appear uniformly colored, and subjects were able to give separate saturation reports for each of four quadrants of these figures. In the upper and lower quadrants, the vertical and concentric lines have different orientation, and no interaction occurred. But in the left and right quadrants, where vertical and concentric lines have similar orientation, saturation was increased on the left (where the CAEs were additive) and decreased on the right (where they were subtractive). Moreover, Broerse et al. (1994) recognized that their results raised doubts about the independence of CAEs established with static polar and Cartesian gratings.

Thus, the case for global factors is now in a situation similar to that faced in the mid-1970s by White and Riggs's arguments for curvature-dependent CAEs; those CAEs were also said not to be entirely explainable in terms of local line orientation (Riggs, 1973, 1974; White \& Riggs, 1974). As noted above, these claims drew a sequence of experimental rejoinders (Crassini \& Over, 1975; MacKay \& MacKay, 1974; Sigel \& Nachmias, 1975; Stromeyer, 1974a), all concluding that curvaturecontingent aftereffects actually behave according to predictions from local line orientation. The similarity between that situation and the present one was foreshadowed by
Crassini and Over (1975), who perceived that CAEs might be expected from curve patterns not only on the basis of local curvature detectors (Riggs's hypothesis) but also on the basis of global processing.

\begin{abstract}
MacKay and MacKay (1974) have shown that curvaturespecific color aftereffects are not induced when the subject has made pursuit movements along the chord of the adaptation arc during the inspection period. This condition should yield aftereffects if curvature is processed on a global basis, but not if visual analysis is undertaken with reference to local orientation information. (Crassini \& Over, 1975, p. 403, italics added)
\end{abstract}

Yet the MacKays had been forced to conclude that if there is any curvature-specific effect "it must ... be weaker by at least an order of magnitude than those attributable to simple orientational adaptation" (MacKay \& MacKay 1974, p. 1286).

The present experiment suggests a similar conclusion for the role of global factors in generating CAEs. If there is such a role, it must be considerably weaker than the role played by local orientation contingencies. It should be remembered, however, that this experiment maintained the central fixation conditions of the original Emerson et al. (1985) study. These conditions favor local factors by keeping the induction patterns in about the same position on the retina. In studies that have systematically varied fixation during induction with polar gratings (Broerse \& O'Shea, 1995; Dodwell \& O'Shea, 1987; Yasuda, 1978), the resulting CAEs have been reduced in magnitude but have not entirely disappeared. Further study may yet provide convincing evidence of a role played by global factors, upholding the view of Dodwell and Humphrey (1990) that local orientation contingencies do not tell the whole story.

\section{REFERENCES}

Allan, L. G., \& Siegel, S. (1986). McCollough effects as conditioned responses: Reply to Skowbo. Psychological Bulletin, 100, 388-393.

Allan, L. G., \& SiEgel, S. (1993). McCollough effects as conditioned responses: Reply to Dodwell and Humphrey. Psychological Review. 100, 342-346.

Allan, L. G., \& Siegel, S. (1997a). Assessing a new analysis of contingent color aftereffects. Cognition, 64, 207-222.

AlLAN, L. G., \& SiEgEL, S. (1997b). Contingent color aftereffects: Reassessing old conclusions. Perception \& Psychophysics, 59, 129-141.

Allan, L. G., Siegel, S., Collins, J. C., \& MacQueen, G. M. (1989). Color aftereffect contingent on text. Perception \& Psychophysics, 46, 105-113.

Allan, L. G., Siegel, S., \& Linders, L. M. (1992). Cue-contingent adaptation to color. Learning \& Motivation. 23, 288-305.

BARLOW, H. B. (1990). A theory about the functional role and synaptic mechanism of visual after-effects. In C. Blakemore (Ed.), Vision: Coding and efficiency (chap. 32, pp. 363-375). Cambridge: Cambridge University Press.

BEDFORD, F. L. (1995). Constraints on perceptual learning: Objects and dimensions. Cognition, 54, 253-297.

BEDFORD, F. L. (1997). Are long-term changes to perception explained by Pavlovian associations or perceptual learning theory? Cognition, 64, 221-230.

Breitmeyer, B. G., \& CoOPER, L. A. (1972). Frequency-specific color adaptation in the human visual system. Perception \& Psychophysics, 11, 95-96.

Broerse, J., \& Grimbeek, P. (1994). Eye movements and the associa- 
tive basis of contingent aftereffects: A comment on Siegel, Allan, and Eissenberg (1992). Journal of Experimental Psychology: General, 123, $81-85$.

BroERSE, J., \& O'SHEA, R. P. (1995). Local and global factors in spatiallycontingent coloured aftereffects. Vision Research, 35, 207-226.

Broerse, J., Shaw, C., Dodwell, P. C., \& Muir, D. (1994). Colored aftereffects contingent upon global transformations? Spatial Vision, 8, 95-117.

Broerse, J., Vladusich, T., \& O'Shea, R. P. (1999). Colour at edges and colour spreading in McCollough effects. Vision Research, 39, 1350-1320.

Cavill, J., \& Robinson, J. O. (1976). A color aftereffect contingent on complex pattern features. Perception \& Psychophysics, 19, 454-459.

Crassini, B., \& Over, R. (1975). Curvature-specific color aftereffects. Perception \& Psychophysics, 17, 398-404.

Dodwell, P. C., \& Humphrey, G. K. (1990). A functional theory of the McCollough effect. Psychological Review, 97, 78-89.

DODWELL, P. C., \& O'ShEA, R. (1987). Global factors generate the McCollough effect. Vision Research, 27, 569-580.

ELLIS, S. R. (1977). Orientation selectivity of the McCollough effect: Analysis by equivalent contrast transformation. Perception \& Psychophysics, 22, 539-544.

Emerson, V. F., Humphrey, G. K., \& Dodwell, P. C. (1985). Colored aftereffects contingent on patterns generated by Lie transformation groups. Perception \& Psychophysics, 37, 155-162.

ForeIt, K. G., \& AmBLER, B. A. (1978). Induction of the McCollough effect I: Figural variables. Perception \& Psychophysics, 24, 295-302.

Gallant, J. L., Connor, C. E., Rakshit, S., Lewis, J. W., \& van Essen, D. C. (1996). Neural responses to polar, hyperbolic, and Cartesian gratings in area V4 of the macaque monkey. Journal of Neurophysiology, 76, 2718-2739.

Held, R., \& Shattuck, S. (1971). Color-and edge-sensitive channels in the human visual system: Tuning for orientation. Science, 174, 314-316.

Hubel, D. H., \& WiEsEL, T. N. (1962). Receptive fields, binocular interaction and functional architecture in the cat's visual cortex. Journal of Physiology, 160, 106-154.

Humphrey, G. K. (1998). The McCollough effect: Misperception and reality. In V. Walsh \& J. Kulikowski (Eds.), Perceptual constancy: Why things look as they do (pp. 31-68). Cambridge: Cambridge University Press.

Humphrey, G. K., Herbert, A. M., Symons, L. A., \& Kara, S. (1994). McCollough effect to "form": A local phenomenon. Journal of Experimental Psychology: General, 123, 86-90.

Humphrey, G. K., Skowbo, D., Symons, L. A., Herbert, A. M., \& Grant, C. L. (1994). Text-contingent color aftereffects: A reexamination. Perception \& Psychophysics, 56, 405-413.

Jameson, D., Hurvich, L. M., \& VARner, D. (1979). Receptoral and postreceptoral visual processes in recovery from chromatic adaptation. Proceedings of the National Academy of Sciences, 76, 3034-3038.

KOHLER, I. (1951). Über Aufbau und Wandlungen der Wahrnehmungswelt; insbesondere über bedingte Empfindungen. Österreichische Akademie der Wissenschaften, Sitzungsberichte, Philosophischhistorische Klasse, 227, 1-118. Available in English translation (1964): The formation and transformation of the perceptual world (H. Fiss, trans.). New York: International Universities Press.

Lovegrove, W. J., \& Over, R. (1972). Color adaptation of spatial frequency detectors in the human visual system. Science, 176, 541-543.

MACKAY, D. M., \& MACKAY, V. (1973). Orientation-sensitive aftereffects of dichoptically presented colour and form. Nature, 242, 477479.

MACKAY, D. M., \& MACKAY, V. (1974). Do curvature-contingent chromatic aftereffects require detectors for curvature? Vision Research, 14, 1285-1287.

McCollough, C. (1965). Color adaptation of edge detectors in the human visual system. Science, 149, 1115-1116.

Mikaelian, H. H. (1980). Effective luminance contrast as a parameter in contingent aftereffects. Perception \& Psychophysics, 27, 531-536.

Murch, G. (1976). Classical conditioning of the McCollough effect: Temporal parameters. Vision Research, 16, 615-619.
PiePER, W. (1995). Color adaptation of edge-detectors is different from color adaptation of stripe-detectors. Perception, 24(Suppl.), 63.

RIGGS, L. A. (1973). Curvature as a feature of pattern vision. Science, 181, 1070-1072.

RigGs, L. A. (1974). Note following "Curvature detectors in human vision?" Science, 184, 1200-1201.

Riggs, L. A., White, K. D., \& Eimas, P. D. (1974). Establishment and decay of orientation-contingent aftereffects of color. Perception \& Psychophysics, 16, 535-542.

Siegel, S., \& Allan, L. G. (1992). Pairings in learning and perception: Pavlovian conditioning and contingent aftereffects. In D. L. Medin (Ed.), Psychology of learning and motivation (Vol. 28, pp. 127-160). San Diego: Academic Press.

Siegel, S., Allan, L. G., \& Eissenberg, T. (1992). The associative basis of contingent color aftereffects. Journal of Experimental Psychology: General, 121, 79-94.

Siegel, S., Allan, L. G., \& Eissenberg, T. (1994). Scanning and formcontingent color aftereffects. Journal of Experimental Psychology: General. 123, 91-94.

Sigel, C., \& NAChmiAs, J. (1975). A re-evaluation of curvature-specific chromatic aftereffects. Vision Research, 15, 829-836.

STROMEYER, C. F. (1974a). Curvature detectors in human vision? Science, 184, 1199-1200.

STROMEYER, C. F. (1974b). Rod signals in higher color mechanisms: The McCollough color aftereffect observed in scotopic illumination. In H. R. Moskowitz, B. Scharf, \& J. C. Stevens (Eds.), Sensation and measurement: Papers in honor of S. S. Stevens (pp. 97-142). Dordrecht: D. Reidel.

Stromeyer, C. F., \& Dawson, B. M. (1978). Form-colour aftereffects: Selectivity to local luminance contrast. Perception, 7, 407-415.

VIOLA, M. M. (1966). Color adaptation contingent upon the geometry of the inducing stimulus. Unpublished honors thesis, Smith College, Northampton, MA.

WATANABE, T. (1995). Orientation and color processing for partially occluded objects. Vision Research, 35, 647-655

WhITE, K. D. (1976). Luminance as a parameter in estabiishment and testing of the McCollough effect. Vision Research, 16, 297-302.

White, K. D., Petry, H. M., Riggs, L. A., \& Miller, J. (1978). Binocular interactions during establishment of McCollough effects. Vision Research, 18, 1201-1215.

White, K. D., \& RigGs, L. A. (1974). Angle-contingent color aftereffects. Vision Research, 14, 1147-1154.

YASUDA, K. (1978). Color aftereffects contingent on MacKay complementary regular patterns. Japanese Psychological Research, 20, $115-$ 123.

\section{NOTES}

1. Experiments requiring observers to shift fixation during induction with polar patterns might give such evidence, provided it can be shown that observers see different colors when fixating horizontal lines in a radial test pattern than when fixating approximately horizontal lines in a concentric pattern. No such finding has been reported. Using the Emerson et al. (1985) procedure, Dodwell and O'Shea (1987) required their observers to shift fixation in a systematic way during successive inductions with two grating pairs (Figures $l a$ and $l b$ ), and evidence of both polar and Cartesian CAEs was still obtained. However, Broerse and O'Shea (1995) have argued that the pattern of fixation shifts did not rule out an explanation in terms of local line orientation.

2 . Note that the coloring books contained only outlines, not copies of the actual test patterns (as in Emerson et al., 1985). Recording one's responses on a booklet page entails shifting fixation, and it was thought that CAEs elicited by patterns on the response page could confuse an observer who is trying to record what has been seen with central fixation on a more distant test pattern.

(Manuscript received June 25, 1998; revision accepted for publication December 21,1998 .) 